feelings prior to onset of convulsive attacks; 2) normal neurologic exam and EEG: 3) family history of deafness, cardiac arrhythmia, or unexpected sudden death; and 4) lack of response to antiepileptic drugs. Familial, autosomal recessive or dominant, and acquired types of prolonged QT interval are recognized. The mortality may be as high as $70 \%$ if unrecognized and untreated.

\title{
VIDEO GAME-INDUCED SEIZURES
}

Fifteen patients, ages 9 to 15 years, who experienced epileptic seizures while playing video games are reported from St Thomas's Hospital, London, UK. An additional 20 patients in 12 reports in the literature are reviewed, and 3 further patients are described in an addendum. The majority had the first seizure as a result of the video game. Seizure patterns were generalized tonic clonic in two thirds; some had absence and $30 \%$ had juvenile myoclonic epilepsy. Photosensitivity occurred in $70 \%$, while excitement, fatigue, sleep deprivation, and cognitive processing were important precipitants in others. Partial, mainly occipital, seizures occurred in 29\%. Management was individualized, and AEDs were not always necessary. (Ferrie CD et al. Video game induced seizures. I Neurol Neurosurg Psychiatry Aug 1994;57:925-931). (Respond: Dr CD Ferrie, Dept Clinical Neurophysiology and Epilepsy, St Thomas's Hospital, London SE1 7EH, UK).

COMMENT. Video game seizures are reflex epilepsies, generalized or partial, and a feature of various idiopathic epileptic syndromes. Both photic and non-photic precipitants are involved. The avoidance of the precipitant may prevent the progression of minor absences, jerks, or visual phenomena to a generalized tonic clonic epilepsy.

See Ped Neur Briefs April 1994, p 28, for a previous report of 10 patients seen at the University of Washington, Seattle, and a review of 20 cases cited in the literature. The comment that video game related seizures are more common than previously recognized appears to be confirmed.

\section{ANTIEPILEPTIC DRUGS}

\section{CEREBELLAR ATROPHY WITH PHENYTOIN AND EPILEPSY}

Cerebellar size measured by MRI was studied in a group of 36 adults (21 to 54 years, mean age 34 years) with intractable partial epilepsy treated with phenytoin longer than 4 years at the Epilepsy Center of the Long Island Jewish Medical Center, New Hyde Park, NY. Patients with IQ $<70$, ethanol abuse, status epilepticus, and neurodegenerative disorders were excluded. Measurements were compared to a group of control patients examined because of headache or dizziness. Mean duration of phenytoin exposure was 14 years (range, 4 to 30 years). Mean maximum dosage was $450 \mathrm{mg}$ daily (range, 300 to $700 \mathrm{mg}$ ). All patients had received various AEDs other than phenytoin. Moderate to severe cerebellar atrophy was found in $9(25 \%)$ patients and mild atrophy in 12 (33\%). The MRI was normal in 15 (42\%) phenytoin exposed patients and in 33 (94\%) controls. A correlation between cerebellar atrophy ratings and variables reflective of seizure severity or degree of phenytoin exposure could not be demonstrated. (Ney GC et al. Cerebellar atrophy in patients with long-term phenytoin exposure and epilepsy. Arch Neurol Aug 
1994;51:767-771). (Reprints: Dr Ney, Dept Neurology, EEG Laboratory, Long Island Jewish Medical Center, New Hyde Park, NY 11042).

COMMENT. The exact cause of the cerebellar atrophy was not determined. The partial seizures, the phenytoin, or both factors were involved. Other known causes of cerebellar atrophy had been excluded. Monitoring the serum phenytoin may have provided a correlation between MRI ratings of atrophy and the possible effects of a chronic level of toxicity. Long-term treatment with phenytoin is generally safe provided optimal therapeutic levels are maintained. Current emphasis on monotherapy may lead to dosage increments above acceptable levels, with the attendant risk of a chronic subtle ataxia, especially in patients with refractory epilepsies.

\section{APLASTIC ANEMIA WITH ETHOSUXIMIDE}

An 8-year-old girl who developed aplastic anemia after 8 months ethosuximide therapy for absence seizures is reported from the Children's Medical College of Virginia, Richmond, VA. Blood counts and liver enzymes had been monitored 3 months before admission and were normal. She presented with fatigue, headache, streptococcal pharyngitis, hematuria, bruising, and petechiae. Allogeneic bone marrow transplantation was required and the child recovered. Without further AED therapy she has only occasional "staring spells" and the EEG is normal. A total of 8 cases of ethosuximide-associated aplastic anemia have been reported, and 5 died. (Massey GV, Myer EC et al. Aplastic anemia following therapy for absence seizures with ethosuximide. Pediatr Neurol July 1994;11:59-61). (Respond: Dr Massey, PO Box 980121, MCV Station, Richmond, VA 23298).

COMMENT. Ethosuximide-related aplastic anemia is rare but has a high mortality. Monthly blood counts have been recommended, but their predictive value is questioned by the authors. Fever, rash, bruising, and petechiae should certainly require immediate investigation.

\section{HEADACHE}

\section{THE EEG IN CHRONIC HEADACHE EVALUATION}

A retrospective analysis of records of 312 children with chronic headache and review of EEGs in 257 are reported from the Tel Aviv Medical Center and University, Israel. Headache was classified as migraine in 143 (55\%); classic migraine in 12 and common migraine in 121 . The mean age at time of EEG was 9 years. The EEG was normal in $80 \%$. Epileptiform activity occurred in $12 \%$ and slowing in $8 \%$. Response was higher to hyperventilation in non-migraine patients and to photic stimulation in those with migraine. The incidence of epileptic EEGs was $11 \%$ in both migraine and tension type headaches; it was $26 \%$ and significantly higher in 15 children with chronic headache described as "very brief," occurring predominantly in girls, several times a week, and without family history for migraine. Prevalence of epilepsy in families of patients with epileptic EEGs did not differ from the total group. Of six children with epileptiform EEGs who were treated with AEDs, 4 responded and had no further headaches and 2 were not benefited. Of 17 children with focal EEG abnormalities, 9 had head CTs, 1 had an arachnoid cyst, and 3 had sinusitis. The authors conclude that the EEG may be of value in some children 